PROCEEDINGS OF THE

AMERICAN MATHEMATICAL SOCIETY

Volume 139, Number 2, February 2011, Pages 535-545

S 0002-9939(2010)10589-0

Article electronically published on August 18, 2010

\title{
INTEGRALS OF POWERS OF LOGGAMMA
}

TEWODROS AMDEBERHAN, MARK W. COFFEY, OLIVIER ESPINOSA,
CHRISTOPH KOUTSCHAN, DANTE V. MANNA, AND VICTOR H. MOLL

(Communicated by Ken Ono)

Abstract. Properties of the integral of powers of $\log \Gamma(x)$ from 0 to 1 are considered. Analytic evaluations for the first two powers are presented. Empirical evidence for the cubic case is discussed.

\section{INTRODUCTION}

The evaluation of definite integrals is a subject full of interconnections of many parts of mathematics. Since the beginning of Integral Calculus, scientists have developed a large variety of techniques to produce magnificent formulae. A particularly beautiful formula due to J. L. Raabe [12] is

$$
\int_{0}^{1} \log \left(\frac{\Gamma(x+t)}{\sqrt{2 \pi}}\right) d x=t \log t-t, \quad \text { for } t \geq 0
$$

which includes the special case

$$
L_{1}:=\int_{0}^{1} \log \Gamma(x) d x=\log \sqrt{2 \pi} .
$$

Here $\Gamma(x)$ is the gamma function defined by the integral representation

$$
\Gamma(x)=\int_{0}^{\infty} u^{x-1} e^{-u} d u,
$$

for $\operatorname{Re} x>0$. Raabe's formula can be obtained from the Hurwitz zeta function

$$
\zeta(s, q)=\sum_{n=0}^{\infty} \frac{1}{(n+q)^{s}}
$$

via the integral formula

$$
\int_{0}^{1} \zeta(s, q+t) d q=\frac{t^{1-s}}{s-1}
$$

coupled with Lerch's formula

$$
\left.\frac{\partial}{\partial s} \zeta(s, q)\right|_{s=0}=\log \left(\frac{\Gamma(q)}{\sqrt{2 \pi}}\right) .
$$

An interesting extension of these formulas to the $p$-adic gamma function has recently appeared in [3].

Received by the editors February 23, 2010 and, in revised form, March 8, 2010.

2010 Mathematics Subject Classification. Primary 33B15.

Key words and phrases. Integrals, transformations, loggamma, Hurwitz zeta function. 
Two of the current authors have investigated definite integrals involving the Hurwitz zeta function [6, 7. As an unexpected corollary, the formula for the integral of $\log ^{2} \Gamma(x)$,

$$
L_{2}:=\int_{0}^{1} \log ^{2} \Gamma(x) d x=\frac{\gamma^{2}}{12}+\frac{\pi^{2}}{48}+\frac{1}{3} \gamma L_{1}+\frac{4}{3} L_{1}^{2}-\left(\gamma+2 L_{1}\right) \frac{\zeta^{\prime}(2)}{\pi^{2}}+\frac{\zeta^{\prime \prime}(2)}{2 \pi^{2}}
$$

was produced. Here $\gamma$ is Euler's constant defined by

$$
\gamma=\lim _{n \rightarrow \infty} \sum_{k=1}^{n} \frac{1}{k}-\log n .
$$

The natural question addressed here is that of an analytic expression for the family of integrals

$$
L_{n}:=\int_{0}^{1} \log ^{n} \Gamma(x) d x, \quad \text { for } n \in \mathbb{N},
$$

that extends the values of $L_{1}$ and $L_{2}$ given above. Section 2 presents a direct approach to the evaluation of $L_{1}$, very close in spirit to the original proof given by Raabe. The proof employs only elementary properties of the gamma function. Section 3 contains a new proof of the value of $L_{2}$ based on the Fourier series expansion of $\log \Gamma(x)$. An expression for $L_{3}$ remains an open question. The quest for such an expression is connected to a special kind of multiple zeta values known as Tornheim sums. The study of their relation with the value of $L_{3}$ was begun in [8, 9.

Section 4 discusses the integrals

$$
S_{n}=(-1)^{n} \int_{0}^{1} \log ^{n}(\sin \pi x) d x
$$

that appear in the evaluation of $L_{2}$. A notion of weight is introduced and a recurrence for this family shows directly that $S_{n}$ is a homogeneous form. The study of the loggamma integrals considered here has been motivated by our conjecture that $L_{n}$ is a homogeneous form of weight $n$. This remains open for $n \geq 3$.

\section{A Riemann sum approach to the evaluation of $L_{1}$}

In this section we present an elementary evaluation of the formula for $L_{1}$. This was originally obtained by J. L. Raabe [12] and it appears as entry 6.441.2 in the classical table [10].

Theorem 2.1. The integral $L_{1}$ is given by

$$
\int_{0}^{1} \log \Gamma(x) d x=\log \sqrt{2 \pi} .
$$

Proof. Partition the interval $[0,1]$ into $n$ subintervals of length $1 / n$ to produce

$$
\int_{0}^{1} \log \Gamma(x) d x=\lim _{n \rightarrow \infty} \sum_{k=1}^{n} \frac{1}{n} \log \Gamma\left(\frac{k}{n}\right) .
$$


On the other hand, assuming $n$ is even,

$$
\begin{aligned}
\frac{1}{n} \sum_{k=1}^{n} \log \Gamma\left(\frac{k}{n}\right) & =\frac{1}{n} \log \left(\prod_{k=1}^{n} \Gamma\left(\frac{k}{n}\right)\right) \\
& =\frac{1}{n} \log \left(\prod_{k=1}^{n / 2} \Gamma\left(\frac{k}{n}\right) \Gamma\left(1-\frac{k}{n}\right)\right) \\
& =\frac{1}{n} \log \left(\prod_{k=1}^{n / 2} \frac{\pi}{\sin (\pi k / n)}\right) \\
& =\log \sqrt{\pi}-\log \left(\prod_{k=1}^{n / 2} \sin (\pi k / n)\right)^{1 / n} .
\end{aligned}
$$

The reflection formula $\Gamma(x) \Gamma(1-x)=\pi / \sin \pi x$ for the gamma function has been employed in the third line.

The classical trigonometric identity

$$
\prod_{k=1}^{n-1} \sin \left(\frac{\pi k}{n}\right)=\frac{n}{2^{n-1}}
$$

now yields

$$
\frac{1}{n} \sum_{k=1}^{n} \log \Gamma\left(\frac{k}{n}\right)=\log \left(\frac{\sqrt{2 \pi}}{(2 n)^{1 / 2 n}}\right) .
$$

Let $n \rightarrow \infty$ to obtain the result. The case $n$ odd is treated similarly.

\section{The evaluation of $L_{2}$}

The expression for $L_{2}$ given in (1.7) was obtained in [6] using integrals involving the Hurwitz zeta function $\zeta(z, s)$, defined in (1.4). Differentiate the identity

$$
\int_{0}^{1} \zeta\left(z^{\prime}, x\right) \zeta(z, x) d x=\frac{2 \Gamma(1-z) \Gamma\left(1-z^{\prime}\right)}{(2 \pi)^{2-z-z^{\prime}}} \zeta\left(2-z-z^{\prime}\right) \cos \left(\frac{\pi\left(z-z^{\prime}\right)}{2}\right)
$$

with respect to $z$ and $z^{\prime}$ and then set $z=z^{\prime}=0$. The formula of Lerch (see [13], page 271),

$$
\left.\frac{d}{d z} \zeta(z, x)\right|_{z=0}=\log \Gamma(x)-\log \sqrt{2 \pi}
$$

produces the result.

In this section we provide a new proof of (1.7) based on the Fourier expansion of $\log \Gamma(x)$ :

$$
\begin{aligned}
\log \Gamma(x) & =L_{1}-\frac{1}{2} \log (2 \sin \pi x)+\frac{1}{2}\left(\gamma+2 L_{1}\right)(1-2 x) \\
& +\frac{1}{\pi} \sum_{k=1}^{\infty} \frac{\log k}{k} \sin 2 \pi k x .
\end{aligned}
$$

This expansion was given by E. Kummer [11; the reader will find a detailed proof in [1]. 
Define

$$
\begin{aligned}
& g(x)=L_{1}-\frac{1}{2} \log (2 \sin \pi x)+\frac{1}{2}\left(\gamma+2 L_{1}\right)(1-2 x) \\
& s(x)=\frac{1}{\pi} \sum_{k=1}^{\infty} \frac{\log k}{k} \sin 2 \pi k x
\end{aligned}
$$

so that

$$
L_{2}=\int_{0}^{1} s^{2}(x) d x+2 \int_{0}^{1} s(x) g(x) d x+\int_{0}^{1} g^{2}(x) d x .
$$

Each term in this sum is now considered separately.

First term. The orthogonality of the trigonometric terms on $[0,1]$ yields

$$
\int_{0}^{1} s^{2}(x) d x=\frac{1}{\pi^{2}} \sum_{k_{1}, k_{2}} \frac{\log k_{1}}{k_{1}} \frac{\log k_{2}}{k_{2}} \int_{0}^{1} \sin \left(2 \pi k_{1} x\right) \sin \left(2 \pi k_{2} x\right) d x=\frac{1}{\pi^{2}} \sum_{k} \frac{\log ^{2} k}{k^{2}} .
$$

Therefore $\int_{0}^{1} s^{2}(x) d x=\zeta^{\prime \prime}(2) / 2 \pi^{2}$ using $\sum_{k=1}^{\infty} \frac{\log ^{2} k}{k^{2}}=\zeta^{\prime \prime}(2)$.

Second term. The "cross term" in 3.5 reduces to

$$
\begin{aligned}
2 \int_{0}^{1} g(x) s(x) d x & =-\frac{1}{\pi} \sum_{k=1}^{\infty} \frac{\log k}{k} \int_{0}^{1} \sin (2 \pi k x) \log (2 \sin \pi x) d x \\
& -\frac{2\left(\gamma+2 L_{1}\right)}{\pi} \sum_{k=1}^{\infty} \frac{\log k}{k} \int_{0}^{1} x \sin (2 \pi k x) d x
\end{aligned}
$$

in view of the vanishing of $\int_{0}^{1} \sin (2 \pi k x) d x=0$, for $k \geq 1$. Integration by parts yields $\int_{0}^{1} x \sin (2 \pi k x) d x=-\frac{1}{2 \pi k}$, converting the last series into $\sum_{k=1}^{\infty} \frac{\log k}{k^{2}}=-\zeta^{\prime}(2)$.

The evaluation $\int_{0}^{1} \sin (2 \pi k x) \log (2 \sin \pi x) d x=0$ appears as 4.384 .1 in [10]. It follows that $\int_{0}^{1} g(x) s(x) d x=-\frac{\zeta^{\prime}(2)}{2 \pi^{2}}(\gamma+\log 2 \pi)$.

Third term. The last term in (3.5) is $\int_{0}^{1} g^{2}(x) d x=L_{1}^{2}+\frac{\pi^{2}}{48}+\frac{1}{12}\left(\gamma+2 L_{1}\right)^{2}$, where we have employed

$$
\int_{0}^{1} \log (2 \sin \pi x) d x=\int_{0}^{1}(1-2 x) \log (2 \sin \pi x) d x=0
$$

and

$$
\int_{0}^{1} \log ^{2}(2 \sin \pi x) d x=\frac{\pi^{2}}{12}
$$

The second integral in (3.6) is seen to vanish by using the change of variables $t=1-x$. The evaluation (3.7) is proven in Section 4. Every term in (3.5) has been evaluated, confirming (1.7). 
Note 3.1. A second proof of (1.7) can be obtained from the Fourier expansion

$$
\log \Gamma(x)=a_{0}+\sum_{n=1}^{\infty} a_{n} \cos (2 \pi n x)+\sum_{n=1}^{\infty} b_{n} \sin (2 \pi n x),
$$

with $a_{0}=L_{1}, a_{n}=\frac{1}{2 n}$ and $b_{n}=\frac{A+\log n}{\pi n}$, with $A=\gamma+2 L_{1}$. This appears in [6] (formulas (6.3) and (6.4)) and it follows directly from entries 6.443.1 and 6.443.3 in [10]. Parseval's identity gives

$$
L_{2}=a_{0}^{2}+\frac{1}{2} \sum_{n=1}^{\infty} a_{n}^{2}+\frac{1}{2} \sum_{n=1}^{\infty} b_{n}^{2}
$$

which produces (1.7).

\section{A FAMILY OF LOG-TRIGONOMETRIC INTEGRALS}

This section considers the family of integrals

$$
S_{k}=(-1)^{k} \int_{0}^{1} \log ^{k}(\sin \pi x) d x
$$

that appeared in the special cases $k=1$ and $k=2$ in the evaluation of $L_{2}$ given in Section 3. These integrals were analyzed in [2], where the value

$$
S_{k}=\left.\frac{(-1)^{k}}{\sqrt{\pi} 2^{k}}\left(\frac{d}{d \alpha}\right)^{k} \frac{\Gamma\left(\alpha+\frac{1}{2}\right)}{\Gamma(\alpha+1)}\right|_{\alpha=0}
$$

was employed to produce the exponential generating function

$$
\sum_{k=0}^{\infty} S_{k} \frac{x^{k}}{k !}=\frac{1}{\sqrt{\pi}} \frac{\Gamma\left(\frac{1-x}{2}\right)}{\Gamma\left(1-\frac{x}{2}\right)} .
$$

From there, the author derived the recurrence

$$
S_{k+1}=S_{k} \log 2+\sum_{j=1}^{k}\left(1-2^{-j}\right) \zeta(j+1) \frac{k !}{(k-j) !} S_{k-j} .
$$

Note 4.1. The initial condition for (4.4) is $S_{1}=\log 2$. This result, due to Euler, appeared in detail in [5], page 182. The value $S_{2}=\pi^{2} / 12+\log ^{2} 2$ is now obtained from the recurrence. These two integrals appear in [10] as 4.241.7 and 4.261.9 respectively. The next two values are

$$
S_{3}=\frac{1}{4} \pi^{2} \log 2+\log ^{3} 2+\frac{3}{2} \zeta(3)
$$

and

$$
S_{4}=\frac{19 \pi^{4}}{240}+\frac{1}{2} \pi^{2} \log ^{2} 2+\log ^{4} 2+6 \log 2 \zeta(3) .
$$

These values do not appear in [10].

Note 4.2. Certain families of integrals can be transformed into homogeneous polynomials by replacing the real numbers appearing in their evaluation by variables. Each number $x$ is provided a weight $w(x)$ and at the moment this assignation is completely empirical. For example, introduce the variables

$$
z_{0}=\log 2 \text { and } z_{1}=\pi,
$$


and

$$
z_{j}=\zeta(j)^{1 / j}
$$

Therefore, the number $\zeta(j)=z_{j}^{j}$ has weight $w(\zeta(j))=j$. The weight satisfies $w(a b)=w(a)+w(b)$. Therefore the weights to $\pi$ and $\zeta(j)$ described above produce the consistent assignment of weight $2 m$ to both sides of the equation

$$
\zeta(2 m)=\frac{2^{2 m-1}\left|B_{2 m}\right|}{(2 m) !} \pi^{2 m} .
$$

Rational numbers have weight 0 .

The integrals $S_{k}$ are now expressed as

$$
\begin{aligned}
& S_{1}=z_{0}, \\
& S_{2}=z_{0}^{2}+\frac{1}{12} z_{1}^{2}, \\
& S_{3}=\frac{1}{4} z_{0} z_{1}^{2}+z_{0}^{3}+\frac{3}{2} z_{3}^{3}, \\
& S_{4}=\frac{19}{240} z_{1}^{4}+\frac{1}{2} z_{0}^{2} z_{1}^{2}+z_{0}^{4}+6 z_{0} z_{3}^{3} .
\end{aligned}
$$

The recurrence (4.4) gives a direct proof of the next result.

Theorem 4.3. The integral $S_{k}$ gives a homogeneous polynomial of degree $k$.

The integrals $S_{n}$ appear in many interesting situations. For instance, let

$$
\Omega(z)=\frac{4 \Gamma(z)}{z \Gamma^{2}(z / 2)}=\prod_{j=1}^{\infty} \frac{\left(1+\frac{z}{2 j}\right)^{2}}{\left(1+\frac{z}{j}\right)} .
$$

Consider the coefficients $\left\{c_{n}\right\}$ in the Taylor series representation:

$$
\Omega(z)=\sum_{j=0}^{\infty} c_{j} \frac{z^{j}}{j !} .
$$

It has been observed that the expression for $S_{n}$ is given by

$$
S_{n}=H_{n}(\log 2)
$$

where

$$
H_{n}(z)=\sum_{k=0}^{n}(-1)^{k}\left(\begin{array}{l}
n \\
k
\end{array}\right) c_{k} z^{n-k}
$$

\section{A RElated family of integrals}

In this section we consider expressions for the integrals

$$
T_{n, j}=\int_{0}^{1}[\log \Gamma(x)]^{j}[\log \Gamma(1-x)]^{n-j} d x,
$$

for $n \in \mathbb{N}$ and $0 \leq j \leq n$. These integrals are intimately connected to the family $\left\{S_{k}\right\}$ described in Section 4 .

Lemma 5.1. The integrals $T_{n, j}$ satisfy the symmetry rule

$$
T_{n, j}=T_{n, n-j} .
$$

Proof. The change of variables $x \mapsto 1-x$ does it. 
Theorem 5.2. Let $n \in \mathbb{N}$. Then

$$
\sum_{j=0}^{n}\left(\begin{array}{l}
n \\
j
\end{array}\right) T_{n, j}=\sum_{k=0}^{n}\left(\begin{array}{l}
n \\
k
\end{array}\right)(\log \pi)^{n-k} S_{k} .
$$

Proof. Expand the $n$-th power of the logarithm of the reflection formula for the gamma function $\Gamma(x) \Gamma(1-x)=\pi / \sin \pi x$.

Corollary 5.3. The integral $L_{1}$ has the value $\log \sqrt{2 \pi}$.

Proof. The previous theorem yields

$$
T_{1,0}+T_{1,1}=S_{0} \log \pi+S_{1}
$$

Clearly $S_{0}=1$ and $S_{1}=\log 2$ was given in Note 4.1. Applying symmetry $\left(T_{1,0}=\right.$ $\left.T_{1,1}\right)$ gives the result.

Note 5.4. The case $n=2$ of Theorem 5.2 yields

$$
T_{2,2}+T_{2,1}=\frac{1}{2}\left[S_{0} \log ^{2} \pi+2 S_{1} \log \pi+S_{2}\right]
$$

that is,

$$
\int_{0}^{1} \log ^{2} \Gamma(x) d x+\int_{0}^{1} \log \Gamma(x) \log \Gamma(1-x) d x=\frac{1}{24}\left(12 \log ^{2}(2 \pi)+\pi^{2}\right) .
$$

Similarly, $n=3$ gives

$$
\int_{0}^{1} \log ^{3} \Gamma(x) d x+3 \int_{0}^{1} \log ^{2} \Gamma(x) \log \Gamma(1-x) d x=\frac{1}{8}\left(\pi^{2} \log (2 \pi)+4 \log ^{3}(2 \pi)+6 \zeta(3)\right) .
$$

Conjecture 5.5. Assume $\log 2$ and $\log \pi$ are transcendental over $K_{n}=\mathbb{Q}(\zeta(2), \ldots$, $\zeta(n))$. Write $S_{n}$ as

$$
S_{n}=\sum_{j=0}^{n} \alpha_{n, j} \log ^{j} 2
$$

Then the coefficients satisfy

$$
\alpha_{n, i}=\left(\begin{array}{c}
n \\
i
\end{array}\right) \alpha_{n-i, 0}
$$

Note 5.6. It has been observed using Mathematica that the sum on the righthand side of (5.3) is the integral $S_{n}$ after replacing $\log 2$ by $\log 2 \pi$. For example, $S_{2}=\pi^{2} / 12+\log ^{2} 2$ becomes

$$
\frac{\pi^{2}}{12}+(\log 2+\log \pi)^{2}=\frac{\pi^{2}}{12}+\log ^{2} 2+2 \log 2 \log \pi+\log ^{2} \pi .
$$

This is the right-hand side of (5.3) for $n=2$. At the moment, a proof is lacking.

Note 5.7. The recurrence (4.4) shows that $S_{n}$ is a polynomial in $\log 2$ written in the form (5.6). Experimental observations of these coefficients that lead to the conjecture stated above are now recorded.

First: The coefficients $\alpha_{n, j}$ are in the field $K_{n}$. 
Second: Note 5.6 and the assumption that $\log \pi$ is transcendental over $K_{n}$ yield a series of relations among the coefficients $\alpha_{n, j}$. A simple calculation produces

$$
\sum_{j=0}^{n-i} \alpha_{n, i+j}\left(\begin{array}{c}
i+j \\
i
\end{array}\right) \log ^{j} 2=\left(\begin{array}{c}
n \\
i
\end{array}\right) \sum_{j=0}^{n-i} \alpha_{n-i, j} \log ^{j} 2,
$$

for $0 \leq i \leq n$.

The use of these relations is illustrated in a simple case: take $i=n$ to obtain $\alpha_{n, n}=\alpha_{0,0}$. The value $\alpha_{0,0}=1$ now shows that $S_{n}$ is a monic polynomial in $\log 2$. Naturally, this follows directly from (4.4).

Third: The further assumption that $\log 2$ is transcendental over $K_{n}$ produces

$$
\left(\begin{array}{c}
i+j \\
i
\end{array}\right) \alpha_{n, i+j}=\left(\begin{array}{c}
n \\
i
\end{array}\right) \alpha_{n-i, j}
$$

The case $i=0$ yields no information, but $0<i \leq n$ and $j=0$ produce (5.7). Therefore every element of a row in the array $\left\{\alpha_{n, k}: 0 \leq k \leq n, n \geq 0\right\}$, except the first one, is determined by the first column.

The first few terms are given by $\alpha_{1,0}=0, \alpha_{2,0}=\frac{1}{2} \zeta(2), \alpha_{3,0}=\frac{3}{2} \zeta(3)$, $\alpha_{4,0}=\frac{3}{4}\left(\zeta^{2}(2)+7 \zeta(4)\right)$, and $\alpha_{5,0}=\frac{15}{2}(\zeta(2) \zeta(3)+3 \zeta(5))$. It would be of interest to develop an algorithm to determine a priori the values of $\alpha_{m, 0}$ without the use of the recurrence (4.4).

5.1. An experimental observation. Denote by $M_{d}$ the set of all monomials in the variables $z_{1}=\pi, \zeta(3), \zeta(5), \zeta(7), \cdots$ with weight $d$. Then

$$
\alpha_{n, n-j}=\sum_{m \in M_{d}} C(m)(n-d+1)_{d} m
$$

for some rational coefficients $C(m)$ to be determined. Experiments have detected some interesting properties, which will be explored in future work. For example, $C\left(z_{1}^{i_{1}} \zeta(3)^{i_{2}} \zeta(5)^{i_{3}} \ldots\right)=C\left(z_{1}^{i_{1}}\right) C\left(\zeta(3)^{i_{2}}\right) C\left(\zeta(5)^{i_{3}}\right)$ and the base cases can be computed as follows:

$$
C\left(z_{1}\right)=1, C\left(z_{1}^{k}\right)=\sum_{i=1}^{(k-1) / 2} \zeta(2 i) \frac{1-2^{1-2 i}}{k-1} C\left(z_{1}^{k-2 l}\right) \text { and } C\left(\zeta(j)^{i}\right)=\frac{\left(1-2^{1-j}\right)^{i}}{j^{i} i !} .
$$

\section{ANALYTIC EXPRESSIONS FOR $L_{3}$}

Attempts to produce a simple form for $L_{3}$ in terms of known special functions have produced some elaborate ones. The next two formulas represent the type of expressions obtained.

Formula 1. The integral $L_{3}$ is given by

$$
\begin{aligned}
L_{3} & =\frac{3}{16}+\frac{\left(\gamma+2 L_{1}\right)^{2}+\log \sqrt{2}\left(\gamma+2 L_{1}\right)}{4 \pi^{2}} \zeta(3) \\
& +\frac{(\gamma+\log (4 \pi))}{8 \pi^{2}} \zeta^{\prime}(3)+\frac{1}{16 \pi^{2}} \zeta^{\prime \prime}(3)+\frac{\left(\gamma+2 L_{1}\right)}{2 \pi^{2}} \sum_{n} \sum_{k<n} \frac{\log (k)}{n k(n-k)} \\
& +\frac{1}{2 \pi^{2}} \sum_{n} \sum_{k<n} \frac{\log (k) \log (n)}{n k(n-k)}-\frac{1}{4 \pi^{2}} \sum_{n} \sum_{k<n} \frac{\log (k) \log (n)}{n k(n+k)}+3 L_{1} L_{2}-2 L_{1}^{3} .
\end{aligned}
$$


Formula 2. The second expression for $L_{3}$ is given in terms of the functions

$$
T_{ \pm}(z, m)=\sum_{n=1}^{\infty} \frac{G_{m}^{ \pm}(n)}{n^{z}}
$$

where

$$
G_{m}^{ \pm}(n)=\sum_{k=1}^{n-1} \log ^{m} k\left(\frac{1}{k} \mp \frac{1}{n+k}\right)
$$

Define

$$
c_{\gamma, \pi}=\gamma+2 \log \sqrt{2 \pi}
$$

Then

$$
\begin{aligned}
16 \pi^{2} \int_{0}^{1} \log ^{3}\left(\frac{\Gamma(x)}{\sqrt{2 \pi}}\right) d x= & \left(4 c_{\gamma, \pi}^{2}+2 c_{\gamma, \pi} \log 2+3\right) \zeta(3)+2\left(c_{\gamma, \pi}+\log 2\right) \zeta^{\prime}(3) \\
& +\zeta^{\prime \prime}(3)+8 c_{\gamma, \pi} T_{+}(2,1)-8 T_{+}^{\prime}(2,1)+4 T_{-}^{\prime}(2,1) .
\end{aligned}
$$

Expanding the integrand on the left produces $L_{3}$ and other terms containing $L_{1}$ and $L_{2}$. The main challenge is in evaluating the double sums, in terms of known values of special functions.

\section{An Experimental Mathematics approach to $L_{3}$}

The weights introduced in Note 4.2 are now extended to include the Euler constant $\gamma$ defined in (1.8). Therefore $\gamma$ is the desingularization of the harmonic series $\zeta(1)$. The assignment $w(\gamma)=1$ is consistent with the weights given to $\zeta(j)$ for $j \geq 2$. The value $w(\log \pi)=1$ is empirical.

The rule that differentiation increases the weight by 1 is motivated by the example below. The explicit formulas for $L_{1}$ and $L_{2}$ given in (1.2) and (1.7), respectively, motivated the following conjecture.

Conjecture 7.1. The integral $L_{n}$ is a homogeneous form of degree $n$.

This section contains experimental studies conducted in order to decide this conjecture for $n=3$. From the experimental point of view, it is natural to employ methods for finding integer relations; the celebrated PSLQ algorithm is specifically designed for this task, but also lattice reduction algorithms such as LLL can be used. Once we have a rough idea which mathematical constants may appear in the result, we can build a basis by considering certain combinations (products) of these constants.

To recover $L_{2}$, we could start with $\pi, \log 2, \log \pi, \gamma, \zeta^{\prime}(2), \zeta^{\prime \prime}(2)$ and take all products of the form $p q$, where $p$ is a polynomial in $\pi, \log 2, \log \pi, \gamma$ of degree at most 2 , and $q$ is either $1, \zeta^{\prime}(2)$, or $\zeta^{\prime \prime}(2)$. All these products are then homogenized to total degree 2 using the variable $z_{1}=\pi$. The resulting basis consists of 30 elements and LLL needs less than a second to find the correct integer relation (a precision of 70 decimal digits was necessary for that).

However, the integral $L_{3}$ so far resisted this approach. It seems reasonable to include quantities such as $\zeta^{\prime \prime \prime}(2)$ and $\zeta(3)$ into the basis. By considering all combinations of degree 3 the number of basis elements easily exceeds 100-depending on the restrictions that are imposed. Although $L_{3}$ was evaluated to more than 400 digits, no relation could be found. This indicates that either higher precision is needed, or that another mathematical constant enters the game. Similar attempts on $L_{4}$ did not succeed either. 


\section{Asymptotics of $L_{n}$}

High precision numerical evaluation of the integrals $L_{n}$ suggest that $L_{n} \sim n$ ! as $n \rightarrow \infty$. The next theorem makes this behavior more precise.

Theorem 8.1. There exist positive constants $c_{i}$ such that

$$
\frac{L_{n}}{n !} \sim \sum_{i=1}^{\infty} \frac{(-1)^{i+1} c_{i}}{i^{n}}
$$

as $n \rightarrow \infty$. An explicit formula for $c_{i}$ given below shows that the first few terms are $c_{1}=1, c_{2}=\gamma, c_{3}=\frac{1}{2} \zeta(2)+\frac{3}{2} \gamma^{2}$ and $c_{4}=\frac{1}{3}\left(8 \gamma^{3}+\gamma \pi^{2}+\zeta(3)\right)$.

Proof. The integral $L_{n}$ is obtained from the expansion

$$
\log \Gamma(x)=-\log x-\gamma x+\sum_{k=2}^{\infty}(-1)^{k} \frac{\zeta(k)}{k} x^{k},
$$

which appears as entry 8.342.1 in [10]. Define $a_{0}=-\log x, a_{1}=-\gamma$ and $a_{k}=$ $(-1)^{k} \zeta(k) / k$. The expansion of

$$
(\log \Gamma(x))^{n}=\left(a_{0}+a_{1} x+a_{2} x^{2}+\cdots\right)^{n}=\sum_{k=0}^{\infty} b_{k} x^{k}
$$

is organized according to powers of $x$ (treating $a_{0}=-\log x$ as an independent variable). In the computation of the coefficient $b_{N}$ it suffices to consider the sum up to $x^{N}$. The multinomial theorem (4], page 28) gives

$$
\left(a_{0}+a_{1} x+\cdots+a_{N} x^{N}\right)^{n}=\sum_{\substack{i_{0}, i_{1} \cdots, i_{N} \geq 0 \\ i_{0}+i_{1}+\cdots+i_{N}=n}} \frac{n !}{i_{1} ! \cdots i_{N} !} a_{0}^{i_{0}} a_{1}^{i_{1}} \cdots a_{N}^{i_{N}} x^{i_{1}+2 i_{2}+3 i_{3}+\cdots+N i_{N}} .
$$

Integrating $(\log \Gamma(x))^{n}$ from 0 to 1 and using

$$
\int_{0}^{1}(-\log x)^{i} x^{j} d x=\frac{i !}{(j+1)^{i+1}}
$$

yields

$$
L_{n}=n ! \sum_{N=0}^{\infty} \sum_{\begin{array}{c}
i_{0}, i_{1}, \cdots, i_{N} \geq 0 \\
i_{0}+i_{1}+\cdots+i_{N}=n \\
i_{1}+2 i_{2}+\cdots+N i_{N}=N
\end{array}} \frac{a_{1}^{i_{1}} a_{2}^{i_{2}} \cdots a_{N}^{i_{N}}}{i_{1} ! \cdots i_{N} !} \frac{1}{(N+1)^{a_{0}+1}} .
$$

The term $N=0$ has coefficient 1 and to isolate the index $i_{0}$ use, for $1 \leq j \leq N$, the bound $j i_{j} \leq N$ to obtain $n-N H_{N} \leq i_{0} \leq n$, with $H_{N}$ the $N$-th harmonic number. Replacing the values of $a_{i}$ and using the notation $\lambda=n-i_{0}$ yields (8.1) with

$$
c_{N+1}=\sum_{\lambda=1}^{N H_{N}} \sum_{\begin{array}{c}
i_{1}, \cdots, i_{N} \geq 0 \\
i_{1}+\cdots+i_{N}=\lambda \\
i_{1}+2 i_{2}+\cdots+N i_{N}=N
\end{array}} \frac{\gamma^{i_{1}} \zeta(2)^{i_{2}} \cdots \zeta(N)^{i_{N}}}{2^{i_{2}} 3^{i_{3}} \cdots N^{i_{N}} i_{1} ! \cdots i_{N} !} \frac{1}{(N+1)^{\lambda-1}} .
$$

As a final observation, we point out that the sum in $\lambda$ may be terminated at $N$ in view of the inequality $\lambda=i_{1}+\cdots+i_{N} \leq i_{1}+2 i_{2}+\cdots+N i_{N}=N$. 


\section{ACKNOWLEDGEMENTS}

The authors wish to thank Eric Rowland and Charles Knessl for discussions on the asymptotics of $L_{n}$. The last author would like to acknowledge the partial support of NSF-DMS 0713836.

\section{REFERENCES}

[1] G. Andrews, R. Askey, and R. Roy. Special Functions, volume 71 of Encyclopedia of Mathematics and its Applications. Cambridge University Press, New York, 1999. MR 1688958 (2000g:33001)

[2] M. G. Beumer. Some special integrals. Amer. Math. Monthly, 68:645-647, 1961. MR0132150 (24:A1997)

[3] H. Cohen and E. Friedman. Raabe's formula for p-adic gamma and zeta functions. Ann. Inst. Fourier, Grenoble, 58:363-376, 2008. MR2401225 (2009d:11165)

[4] L. Comtet. Advanced Combinatorics. D. Reidel Publishing Co., Dordrecht, Holland, 1974. MR0460128 (57:124)

[5] J. Edwards. A treatise on the Integral Calculus, volume 2, MacMillan, New York, 1922.

[6] O. Espinosa and V. Moll. On some definite integrals involving the Hurwitz zeta function. Part 1. The Ramanujan Journal, 6:159-188, 2002. MR1908196 (2003f:11127)

[7] O. Espinosa and V. Moll. On some definite integrals involving the Hurwitz zeta function. Part 2. The Ramanujan Journal, 6:449-468, 2002. MR2125010 (2005m:11162)

[8] O. Espinosa and V. Moll. The evaluation of Tornheim double sums. Part 1. Journal of Number Theory, 116:200-229, 2006. MR2197867 (2007c:11100)

[9] O. Espinosa and V. Moll. The evaluation of Tornheim double sums. Part 2. The Ramanujan Journal, 22:55-99, 2010. MR2610609

[10] I. S. Gradshteyn and I. M. Ryzhik. Table of Integrals, Series, and Products. Edited by A. Jeffrey and D. Zwillinger. Academic Press, New York, 7th edition, 2007. MR2360010 (2008g:00005)

[11] E. Kummer. Beitrag zur Theorie der Function $\Gamma(x)$. J. Reine Angew. Math., 35:1-4, 1847.

[12] J. L. Raabe. Angenäherte Bestimmung der Factorenfolge $1 \cdot 2 \cdot 3 \cdot 4 \cdot 5 \ldots n=\Gamma(1+n)=$ $\int x^{n} e^{-x} d x$, wenn $n$ eine sehr grosse Zahl ist. J. Reine Angew. Math., 25:146-159, 1840.

[13] E. T. Whittaker and G. N. Watson. Modern Analysis. Cambridge University Press, 1962. MR0178117 (31:2375)

Department of Mathematics, Tulane University, New Orleans, Louisiana 70118

E-mail address: tamdeberhan@math.tulane.edu

Department of Physics, Colorado School of Mines, Golden, Colorado 80401

E-mail address: mcoffey@mines.edu

Departmento de Fisica, Universidad Santa Maria, Valparaiso, Chile

E-mail address: olivier.espinosa@usm.cl

Department of Mathematics, Tulane University, New Orleans, Louisiana 70118

E-mail address: ckoutsch@tulane.edu

Department of Mathematics and Computer Science, Virginia Wesleyan College, NORFOLK, ViRginia 23502

E-mail address: dmanna@vwc.edu

Department of Mathematics, Tulane University, New Orleans, Louisiana 70118

E-mail address: vhm@math.tulane.edu 